\title{
Diagnostic Relevant Visualization of Vascular Structures
}

\author{
Armin Kanitsar ${ }^{1}$, Dominik Fleischmann ${ }^{2}$, Rainer Wegenkittl ${ }^{3}$, and Meister Eduard \\ Gröller $^{1}$ \\ 1 Institute of Computer Graphics and Algorithms, \\ Vienna University of Technology, Vienna, Austria, \\ \{kanitsar,meister\}@cg.tuwien.ac.at \\ 2 Department of Radiology, Stanford University, US, \\ dominik.fleischmann@univie.ac.at \\ 3 TIANI Medgraph, Austria, \\ rainer.wegenkittl@tiani.com
}

Summary. Traditional volume visualization techniques sometimes provide incomplete clinical information needed for applications in medical visualization. In the area of vascular visualization important features such as the lumen of a diseased vessel segment may not be visible. One way to display vascular structures for diagnostic purposes is to generate longitudinal cross-sections in order to show their lumen, wall, and surrounding tissue in a curved plane. Curved planar reformation (CPR) has proven to be an acceptable practical solution. We discuss four different methods to generate CPR images from single vessel segments: Projected CPR, stretched CPR, straightened CPR, and helical CPR. Furthermore we investigate three different methods for displaying vascular trees: Multi-path projected CPR, multi-path stretched CPR, and untangled CPR. The principle concept of each method is discussed and detailed information for the realization is given. In addition the properties, advantages and disadvantages of each method are summarized.

\section{Introduction}

Non-invasive imaging of the vascular system with computed tomography (CT) and magnetic resonance imaging (MRI) has become a well established alternative to invasive intraarterial angiography. CT and MRI provide high-resolution volumetric data sets of the human body. These data, however, may contain many objects of less or no diagnostic interest. This makes volume-rendering (i.e., maximum intensity projection (MIP), ray casting, shaded surface display) without preprocessing often impossible or inaccurate. In addition to that pathological features may superimpose diagnostically relevant information. In the case of a circular vessel wall calcification the true vessel lumen can not be determined by conventional volume rendering.

$C P R$ - Curved Planar Reformation is a way to visualize vascular structures with small diameters. High level information as the vessel's centerline is used to re-sample 
and visualize the data. By this technique the entire tubular structure is displayed within a single image. Vascular abnormalities, i.e., stenoses, occlusions, aneurysms and vessel wall calcifications, are then investigated by physicians. This process is sometimes referred to as Multi Planar Reformation (MPR). However the term MPR is not precise enough, as it is commonly used for planar cross-sections re-sampled from volumetric data. Another known synonym for curved planar reformation is $m e$ dial axis reformation (MAR).

Even though CPR is an established technique in the medical community, the visual properties, advantages, and disadvantages of different types of CPRs have not been specifically addressed in the literature. Understanding this features is essential for the accurate interpretation of the resulting images. One issue of this work is the generation and discussion of properties of different CPR methods.

Traditional CPR techniques allow the investigation of the vessel lumen in a longitudinal section through the central axis of the vessel. However, vascular abnormalities might not be touched by this plane and therefore they do not appear in the generated image. One way to overcome this problem is to rotate the re-sampled plane around the central axis. This results in a set of images to be interpreted by the radiologist. A more comprehensive display of the entire vascular lumen in one representative image is highly desirable. A new visualization method was developed to overcome this limitation.

Another important aspect in computed tomography angiography (CTA) is the efficient visualization of treelike vascular structures using CPR display techniques. Multi-path CPR techniques based on a projective combination of vessel segments provide a spatially coherent display of the vascular anatomy. However, depending on the intersecting plane, parts of the arteries might be superimposed by other arteries. For a detailed inspection of the entire vascular tree different sections through the vessel's central axis have to be re-sampled. In order to have diagnostically valuable results the vessel lumen should be visible within each image. Thus a new technique for unobstructed displaying of an arterial tree is proposed.

Section 2 describes related work in this area. In Section 3 four methods for visualizing vascular segments are presented. Techniques for displaying entire vascular trees are presented in Section 4. Finally the proposed methods are summarized and compared in Section 5.

\section{Related Work}

The most important prerequisite for CPR visualization is an appropriate estimation of the vessel centerline. Latest CT technology, such as multiple detector-array CT, provide high resolution volumetric data sets. Due to the large size of these data sets (up to 1500 transverse cross-sectional images of the abdomen and entire legs), the manual definition of the vessel centerline is no longer an option. In this respect several algorithms $[1,2,3]$ have been developed with different properties concerning reliability, speed and accuracy. 
Avants and Williams [4] present a vessel tracking method consisting of two parts. From user defined seed points a surface expansion is computed based on the eikonal partial differential equation. A minimal cost path is calculated from these regions. From this path a cross-sectional area/radius profile is generated.

He et al. [5] proposed a path extraction method based on a two-dimensional region-growing algorithm with a subsequent shortest path algorithm. The resulting path is refined using the multi-scale medial response. The vascular tree is flattened in a semiautomatic method called Medial Axis Reformation.

Some authors propose to take the central axis as an input for the generation of an abstract vessel model. Abstract vessel models allow fast rendering, as polygonal meshes of low complexity are generated [6]. Furthermore non-photorealistic rendering provides the possibility to emphasize global properties of the vascular tree [7].

Kanitsar et al. [8] compared three methods for CPR generation: Projected CPR, stretched CPR and straightened CPR with respect to three extensions. These extensions have been proposed to overcome the most relevant clinical limitations, i.e., thick CPR, rotating CPR and multi-path CPR.

A comparison of this technique with conventional volume visualization techniques is not the topic of this paper, as such comparisons are already available [9]. Further information about the clinical relevance of the CPR visualization technique can be found in $[10,11,12]$.

\section{Single Vessel CPR Methods}

The goal of CPR visualization is to make a tubular structure visible in its entire length within one single image. To accomplish this goal a-priori information about the tubular structure, notably the object's central axis, is required. Without loss of generality the object's central axis is assumed to be a sequence of points at sub voxel resolution.

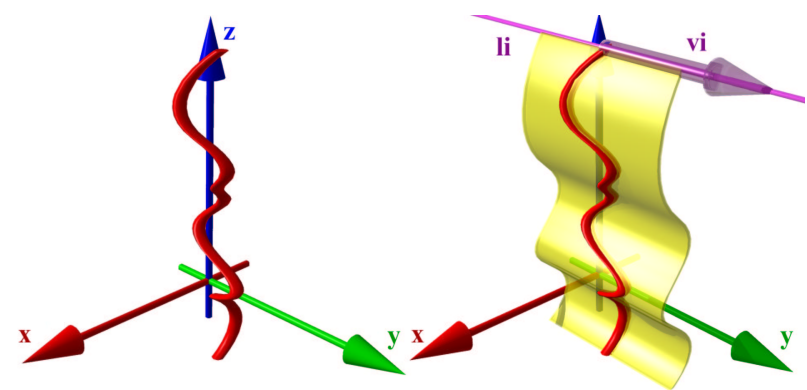

Fig. 1. Principle of the CPR visualization: The vector-of-interest (vi) and the line-of-interest (li) define the re-sampling plane. 
In general the spatial position and shape of the central axis determines which parts of 3D space are visualized. On the left side of Figure 1 the central axis is shown. The re-sampled surface is shown on the right side of Figure 1. As the surface is not well defined by just one curve in 3D, an additional vector vi (vector-of-interest) is introduced. Together with a point from the central axis, the vector-of-interest defines a straight line li (line-of-interest). All voxels touched by this line are taken to resample the volume along the line-of-interest.

Figure 2 illustrates the different CPR methods. The horizontal plane represents the image and the image y-axis as horizontal blue arrow. Corresponding to this axis, the curve in the volumetric data set is sketched by the vertical blue arrow.

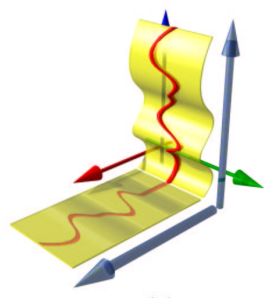

(a)

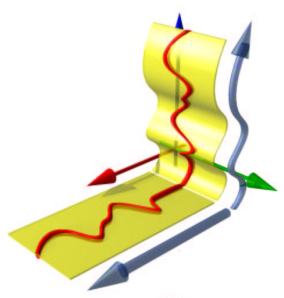

(b)

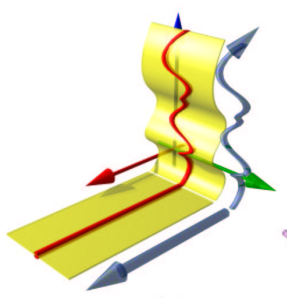

(c)

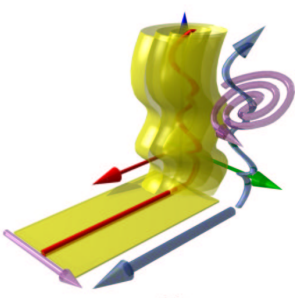

(d)

Fig. 2. CPR generation methods: a) projected, b) stretched, c) straightened, d) helical CPR.

\subsection{Projected CPR}

The projected CPR can be seen as a parallel projection of a data set, taking into account only a thin slice of voxels (see Figure 2a). This slice is defined by the central axis of the tubular structure and the vector-of-interest. We assume the vector-ofinterest to be colinear with thy $y$-axis and apply a parallel projection to a free-form surface along the y-axis.

In particular, for each point of the central axis the line-of-interest is projected to the corresponding line of the image. This relationship is defined by the camera's coordinate system (i.e., the up-vector). If the up-vector of the camera is parallel to the $\mathrm{z}$-axis, the $\mathrm{z}$-coordinate of the line-of-interest is mapped directly to the image. If multiple lines-of-interest project onto the same image area compositing is done using maximum intensity projection (MIP), minimum intensity projection (MinIP), or averaging (AVG). Due to parallel projection the spatial relations are maintained by this method. This helps the observer to perceive the spatial arrangement of the vessels.

The first disadvantage of this method is that structures of higher intensity (i.e., bony structures) still may obscure the structures of interest (i.e., vessels). This situation arises, if parts of the line-of-interest associated with a certain point of the central axis contains bony structures and these parts are projected to an image region 
containing vascular structures from another line-of-interest. The occurrence of such situations heavily depends on the application area. In the case of peripheral vascular structures this case hardly ever arises. However, the visualization of the carotid artery at the level of the skull-base often leads to such situations.

Another disadvantage of the projected CPR method is the distortion of the central axis' length due to parallel projection. Therefore isometry is not preserved.

\subsection{Stretched CPR}

The surface defined by the vessel central axis and the vector-of-interest is curved in one dimension and planar in the other one. Stretching the curved dimension results in a plane showing the tubular structure in it's entirety without overlapping (see Figure $2 b)$. This type of CPR is referred to as stretched CPR.

Processing all points of the central axis successively, the corresponding lines-ofinterest are mapped to the image. This is done by rotating the consecutive point of the central axis around the current line-of-interest. The point is rotated in a way that the resulting plane is coplanar to the viewing plane. Isometry is maintained by this operation as the distance between the two consecutive points is preserved in image space.

Especially we are only interested in the image y-coordinates of the lines-ofinterest. Let's assume point $P_{i}$ to be the last processed point and point $P_{i+1}$ the currently processed point of the central axis. The vector $\mathbf{d}_{i}=\overrightarrow{P_{i} P_{i+1}}$ represents the path direction at position $i$. Furthermore $\mathbf{I}$ is the normalized direction of the line-ofinterest. According to formula (1) the offset $\Delta_{i}$ in image space is.

$$
\Delta_{i}=\sqrt{\left|\mathbf{d}_{i}\right|^{2}-\left(\mathbf{l} \cdot \mathbf{d}_{i}\right)^{2}}
$$

The image position $y_{i+1}$ of the line-of-interest related to point $P_{i+1}$ is given by $y_{i+1}=$ $y_{i}+\Delta_{i}$ where $y_{0}=0$.

The central axis is assumed to be sampled with sub-voxel resolution. Therefore all rows of the image are filled. Introducing a zooming capability requires to interpolate between the lines-of-interest, if necessary.

The generation process of a stretched CPR ensures that other objects do not cover vascular structures. This is one of the key requirements in vessel visualization. The curvature of the tubular structure is still largely maintained by this kind of visualization, thus spatial orientation is still possible for the user.

The main advantage of this CPR type is the preserved isometry. This is important for accurate preoperative planning of endovascular stent-graft treatment of aortic aneurysms. The lengths of normal and abnormal vascular segments need to be determined accurately for sizing an endovascular prosthesis.

\subsection{Straightened CPR}

The third type of curved planar reformation fully straightens the tubular structure (see Figure 2c). This CPR method generates a linear representation of the vessel with varying diameter. 
At each point $P_{i}$ of the central axis the tangent vector $t_{i}$ is calculated. The plane $\varepsilon_{i}$ (cross-section) is defined by $P_{i}$ and $t_{i}$. A local coordinate system is defined by two generating vectors of the plane $\varepsilon_{i}: \overrightarrow{u_{i}}$ and $\overrightarrow{v_{i}}$ whereby $\overrightarrow{u_{i}} \perp \overrightarrow{v_{i}}$. The line-of-interest is defined within the plane $\varepsilon_{i}$ by an angle within the unit circle: the angle-of-interest $\varphi$.

As either $\vec{u}$ or $\vec{v}$ is mapped to the local coordinate system's $\mathrm{x}$-axis, excessive rotation along the central axis may cause undesired artifacts. Methods exist to minimize this effect [13].

In particular it is not necessary to re-sample the entire cross-section from the data set. It is more efficient to do a transformation from the local coordinate system to the global coordinate system. The direction of the line-of-interest $\mathbf{I}_{i}$ is given by formula (2):

$$
\mathbf{l}_{i}=\cos \varphi \cdot \mathbf{u}_{\mathbf{i}}+\sin \varphi \cdot \mathbf{v}_{\mathbf{i}}
$$

The image offset $\Delta_{i}$ for the line-of-interest corresponding to point $P_{i+1}$ equals to the distance from point $P_{i}$ to $P_{i+1}$ :

$$
\Delta_{i}=\left|\overrightarrow{P_{i} P_{i+1}}\right|
$$

The most obvious disadvantage is the lack of spatial orientation. Only short segments of visible side branches of the parent vessels indicate the topographic position of a given arterial segment.

One advantage of this method is the preserved isometry. Furthermore the direct relation between image height and central axis length makes it easy to create linked displays. Whenever the user points to a certain position of the image, the corresponding cross-section is displayed in a separate view. This feature improves on the lack of spatial orientation.

Another advantage is the easy perception of diameter variations. Due to the elimination of the curvature of the central axis the only varying property along the central axis is the diameter.

\subsection{Helical CPR}

The basic idea of helical CPR visualization is to display the volumetric interior of a vessel within one image. To accomplish this, a re-sampling strategy different from the previously discussed CPR methods is introduced. All previous mentioned methods re-sample the data in a linear way defined by the vector $v i$. The vector-of-interest describes the re-sampling direction which is orthogonal to the viewing direction.

The helical CPR method is based on a non-linear re-sampling of the data. The vector-of-interest as generating element for the surface is replaced by a spiral-ofinterest (si) (see Figure 2d). This results in a convoluted surface around the central axis. With a sufficiently small distance between each winding the vessel is intersected several times. Stenoses, calcifications, and occlusions are included in the computed surface. The helical surface is flattened and displayed. 


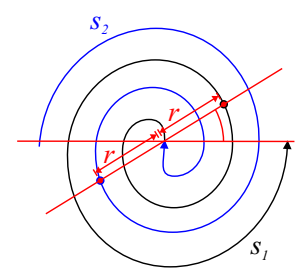

(a)

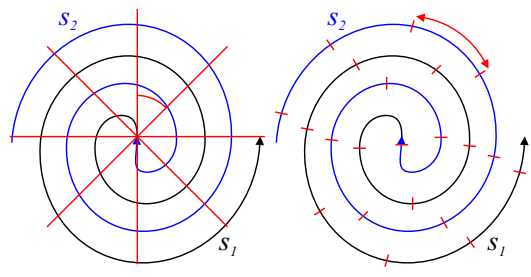

(b)

(c)

Fig. 3. Spiral-of-interest (a), constant angle sampling (b), constant arc-length sampling (c)

\section{Method Description}

Along the central axis of the vessel cross-sections are calculated at an appropriate sampling distance. Within each section a local 2D coordinate system is defined. The center of the cross-section represents the estimated center of the vessel lumen at the corresponding centerline position. Starting from this center point two interleaved spirals $s_{1}$ and $s_{2}$ are computed (see Figure 3a). In order to maintain a uniform sampling of the vessel cross-sections a spiral with constant inter-winding distance is selected. This requirement is satisfied by the Archimedean spiral which can be expressed in a simple equation using polar coordinates $r$ and $\theta$ :

$$
r=a \theta
$$

The transformation of points on the spiral into Cartesian coordinates is straightforward. Thus the computation of a point $X_{s_{1}}$ on $s_{1}$ and a point $X_{s_{2}}$ on $s_{2}$ is performed as follows:

$$
X_{s_{1}}=a \theta\left(\begin{array}{c}
\cos \theta \\
\sin \theta
\end{array}\right) \quad X_{s_{2}}=a \theta\left(\begin{array}{c}
\cos \theta^{\prime} \\
\sin \theta^{\prime}
\end{array}\right) \quad \text { where } \theta^{\prime}=\theta+\pi
$$

For an appropriate sampling of the vessel lumen the parameter $a$ was set to $1 / \pi$. This assures a constant distance of one between the windings of the two interleaved spirals. The computed points $X_{s_{1}}$ and $X_{S_{2}}$ on the spirals are transformed back into volume space and re-sampled.

The center of each scanline in the final CPR image corresponds to the center of the vessel cross-section. Starting from this reference point the image space to the left is filled with data re-sampled by $s_{1}$ and to the right with data from $s_{2}$.

\section{Sampling Strategy}

The current implementation of the helical CPR technique supports two sampling strategies for computing points from the spirals. In the case of constant angle sampling (see Figure 3b) the angle $\theta$ is increased by a constant angle $\omega$ for each point. If constant arc-length sampling is applied (see Figure 3c) for each sampling step a constant distance $\Delta$ on the arc-length of the spiral is covered. 


\section{Constant angle.}

For each point re-sampled from the spiral the generating angle $\theta$ is incremented by a fixed angle $\omega$. Each winding is rendered into an equal sized area in the final image. Therefore the comparably dense sampled area in close vicinity of the vessel center is amplified in image space. The resulting fish-eye zooming effect is achieved at the cost of an increased distortion.

\section{Constant arc-length.}

Given a fixed sampling distance $\Delta$ between two adjacent points on the spiral the increment $\omega$ of the angle $\theta$ is approximated. The increment $\omega$ is defined by the ratio of $\Delta$ and the circumference calculated from the most recent radius. As usually small sampling distances are used the error introduced by this approximation is negligible. The extent of the vessel in the CPR image is directly proportional to the volume of the vessel lumen. Thus large vessels occupy superproportional large parts of the image space.

\section{Vessel Tree CPR Methods}

One substantial disadvantage of CPR visualization for diagnostic purposes is the restriction to only one tubular element. Most clinically relevant 'tubes' are part of a branching, anatomic structure. For instance the peripheral arterial tree begins at the abdominal aorta and branches into the left and right common iliac artery which again branches into internal and external iliac arteries and so on. A comprehensive display of all clinically relevant vessels in one image is highly desirable.

The straight forward approach of simply compositing all calculated CPRs does not produce the desired result. No matter which re-sampling strategy is applied artifacts are always introduced in the generated image. Vascular structures are obscured by bones from other layers. Therefore we propose a new image space driven method for compositing projected or stretched CPRs from multiple central axes.

\subsection{Multi-Path CPR}

The tree of central axes and the volumetric data set is taken as input for the algorithm (Figure 4a). An enhanced z-buffer $b$ of the same size as the image provides space for information entries containing a reference to a path, a reference to a point of this path (point), and depth information. Furthermore each entry contains information about an associated span begin and end. A span represents the part of an image scanline belonging to a certain path segment.

\section{Tree projection.}

The tree (see Figure 4b) is mapped to the buffer $b$ according to the applied CPR method. Figure $4 \mathrm{c}$ shows the entries of the buffer after the CPR projection process. If points of different paths are mapped to the same image position, the one spatially closer to the observer is taken. 


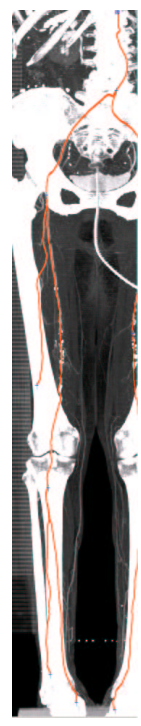

(a)

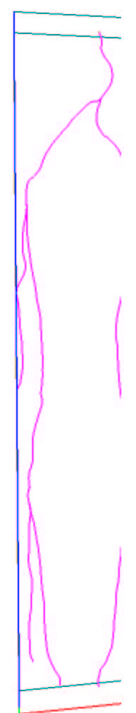

(b)

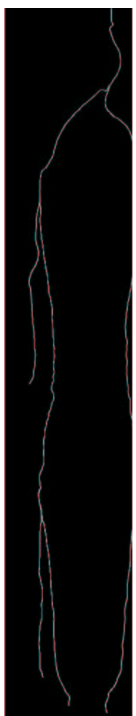

(c)

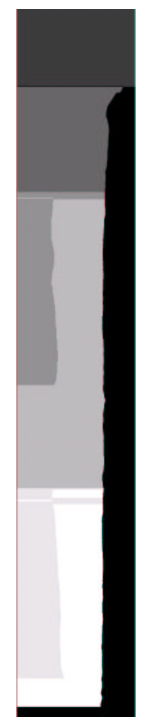

(d)

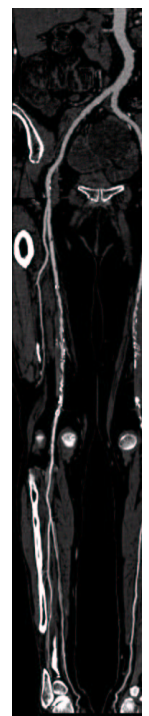

(e)

Fig. 4. Generation of multi-path CPRs.

\section{Buffer traversal.}

In a second step two buffer traversals are needed to determine the length of a span. A span is computed so that the space between neighboring paths within a line is equally divided. The scanline portions at the border of the image are assigned to the leftmost or rightmost path segment respectively (see Figure 4d).

\section{Data re-sampling.}

In the final step the buffer is traversed again. Each filled entry $b[x, y]$ is processed so that the image line $y$ is filled from position $b[x, y]$.begin to $b[x, y]$.end with the data values associated with point $b[x, y]$.point.

This results in a composited CPR through multiple vessel centerlines without overlapping structures, as each tree segment is drawn in a separate image region (see Figure 4e).

A projected multi-path CPR is generated by using the projected CPR method for each vessel segment in the tree projection step. The properties of the projected CPR method also hold true for the projected multi-path CPR.

Similar to the previously mentioned multi-path CPR method the stretched multipath CPR method is generated by using the stretched CPR method for tree projection. In the recursive tree traversal the image position $y_{0}$ of the vessel segment's first line-of-interest has to be handled correctly. 


\subsection{Untangled CPR}

The aim of untangled CPR visualization is to display a vascular tree without overlapping arteries [14]. In order to accomplish this requirement the spatial relations of the projected vessels have to be relaxed. Branching points of the vessel tree are used as pivot points. Rotating the corresponding vessels around these pivot points in image space eliminates vessel overlaps. Keeping the introduced distortions small maintains fast perception and reduces the impact of re-sampling artifacts in the final image. Thus the applied transformations are restricted to the branching points (bifurcations) of the arterial tree only. Additionally these transformations should be appropriate in terms of changing the tree layout and appearance without violating the non-intersection criterion. The non-intersection criterion is defined in a way that two vessel hulls must not intersect at any time.

The input of the algorithm is a tree graph representing the topology of the vascular structure. For each vessel segment the centerline of the vessel is stored as a set of adjacent points at an appropriate sampling distance. In practice it turned out that diameter estimations of vessels are not reliable enough in certain cases. Therefore for the purpose of generality the algorithm does not take diameter information into account. However, the adaptation to this additional information would be straightforward.

\section{Method outline}

The untangled CPR method consists of four main steps. As all untangling calculations are performed in image space the tree graph is first mapped to image space using a stretched CPR projection. In a consecutive step a rotation of each subtree with respect to the non-intersection criterion is performed. Afterwards the image space is partitioned in a way that each vessel obtains those parts of the image space which are closest in scan-line direction. Finally the image is rendered.

\section{Tree projection.}

The vascular tree is mapped to a projection plane parallel to the viewing plane. For two successive points on a vessel path the subsequent point is rotated around an axis defined by the previous point and the vector-of-interest. The rotation is carried out for each point starting from the root of the vascular tree. The result is a stretched vascular tree.

\section{Untangling operation.}

From the projected tree graph in image space the necessary transformations for each node are calculated. This is done by recursively circumscribing the subtrees with vessel hulls. The first pass is bottom up maintaining only the correct transformation of the largest enclosing vessel hull. In a second pass the final transformation for each vessel hull is accumulated top down. 
Image space partitioning.

Before rendering the final image the extent of each vessel segment is cropped in a way that no overlapping image areas remain. This process determines the starting point and the end point of each scanline for rendering.

Rendering.

Each vessel segment is rendered separately. Conceptually the vessel strip is first extracted from the dataset using a stretched CPR mapping. Afterwards the strip is transformed to the position defined by the untangling process. In a further step the strip is clipped according to the space partitioning information. Finally each cropped scanline is rendered into the image.

\section{The vessel hull primitive}

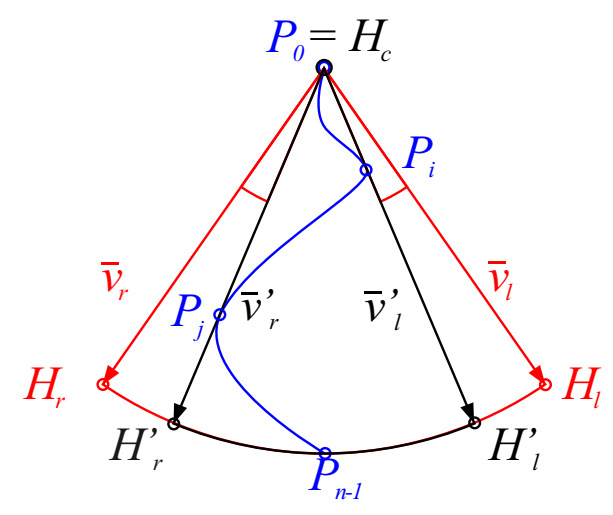

Fig. 5. The vessel hull primitive

The vessel hull is the basic primitive for further intersection tests. It encloses the vessel's centerline in image space as shown in Figure 5. The centerline is given as a set of points $P=\left\{P_{0} . . P_{n-1}\right\}$. A vessel hull is a sector of a circle. The root of a vascular subtree defines the center point $H_{c}$. A matrix $\Re_{H_{c}}$ associated with each center point describes a rotational transformation of the subsequent tree. The points $H_{r}^{\prime}$ and $H_{l}^{\prime}$ result from a conservative estimation of the leftmost and rightmost extent of the enclosing subtree seen from the center point.

The vessel hull encloses the centerline of the vessel thus two neighboring vessels touching each other is not prevented by this primitive. To overcome this situation the vessel hull is enlarged by a small angle $\varepsilon$ as depicted in Figure 5. Depending on the size of the inspected vessels this $\varepsilon$ may be adjusted by the user on the fly. However an $\varepsilon \leq 2^{\circ}$ was found to be appropriate for most tested datasets. If the vessel 
diameter is known at the extremal points $P_{i}$ and $P_{j}$ then $\varepsilon$ can be easily calculated more accurately. The points comprising the $\varepsilon$-tolerance are referred to as $H_{r}$ and $H_{l}$.

Each vessel hull primitive can be described as a tuple of $V=\left\{H_{c}, H_{l}, H_{r}, \Re_{H_{c}}\right\}$ where $\mathbf{v}_{l}={\overrightarrow{H_{c} H_{l}}}_{l}$ and $\mathbf{v}_{r}=\overrightarrow{H_{c} H_{r}}$.

\section{Putting things together}

A rule based approach is applied for combining vessel hulls from various parts of the vascular tree (see Figure 6). The projected vessel tree is approximated by an enclosing hierarchy of vessel hulls. Constructing the vessel hull hierarchy involves several cases which are discussed in the following. A vessel hull created from the vessel centerline is based on case 1 . Neighboring vessel hulls are combined according to case 2. An enclosing vessel hull from two consecutive vessel hulls is created in case 3. The assembling process is done bottom up. This results in a binary tree where each node is represented by a vessel hull circumscribing all subjacent vessel hulls.

\section{Case 1.}

The center point $H_{c}$ is defined by the first point $P_{0}$ of the vessel segment (Figure 6a). The first point on the convex hull of the vessel segment in clockwise orientation is denominated as point $P_{i}$ and in counterclockwise direction as point $P_{j}$. Because of the stretched CPR mapping from volume to image space, point $P_{n-1}$ is the point with maximum distance from $P_{0}$. Thus the radius of the vessel hull is computed as $\left|P_{n-1}-P_{0}\right|$. The vectors $\mathbf{v}_{l}$ and $\mathbf{v}_{r}$ represent the directions from $H_{c}$ to $P_{i}$ and $P_{j}$ respectively. These vectors are scaled according to the radius of the vessel hull. The tolerance angle $\varepsilon$ is incorporated by a rotation with $\Re_{\varepsilon}$ and $\Re_{-\varepsilon}$. Finally $H_{r}$ and $H_{l}$ are computed.

\section{Case 2.}

For the case of two adjacent vessel hull primitives $V^{1}$ and $V^{2}$ (see Figure 6b) the ordering has to be determined. The left vessel hull with respect to $H_{c}=H_{c}^{1}=H_{c}^{2}$ is denominated as $V^{l}$ and the right one as $V^{r}$. If the vessel hull primitives overlap, an untangling angle $\gamma$ is computed from $\mathbf{v}_{l}^{r}$ and $\mathbf{v}_{r}^{l}$ (see Figure 9). From this angle the rotational matrices $\Re_{H_{c}^{r}}$ and $\Re_{H_{c}^{l}}$ are calculated. These matrices define a transformation of the vessel hull primitives $V^{r}$ and $V^{l}$ in a way that the primitives do not overlap anymore. This implies a transformation of the associated vascular subtree (see Figure 9).

The vectors $\mathbf{v}_{l}$ and $\mathbf{v}_{r}$ of the combined vessel hull are computed from the transformed vectors $\mathbf{v}_{l}^{l}$ and $\mathbf{v}_{r}^{r}$. The radius of the enclosing vessel hull is defined by the maximum radius of $V^{1}$ and $V^{2}$. From this information $H_{l}$ and $H_{r}$ is computed. The newly generated vessel hull encloses the non-overlapping underlying vessel hulls. 

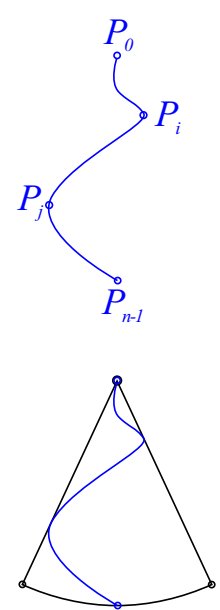

Case I
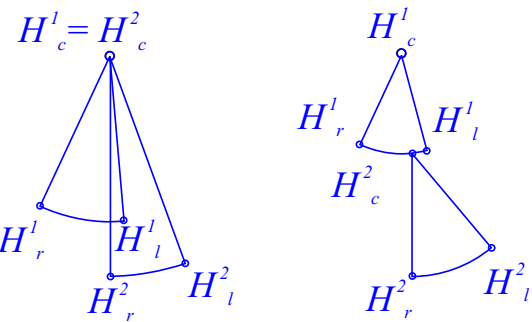

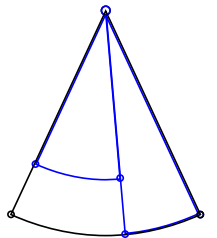

Case II

(b)

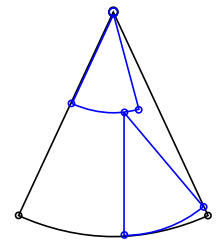

Case III

(c)

Fig. 6. The three vessel hull combination cases.

\section{Case 3.}

The combination of two successive vessel hulls is straightforward. $V^{1}$ is considered to be the predecessor of $V^{2}$ as depicted in Figure $6 c . H_{c}^{1}$ is taken as the new center point $H_{c}$ of the enclosing vessel hull. The direction to the rightmost point of $H_{r}^{1}$ and $H_{r}^{2}$ with respect to $H_{c}$ is considered to be $\mathbf{v}_{r}$. Vector $\mathbf{v}_{l}$ is calculated likewise. The radius of the new vessel hull $V$ is defined by the maximum distance from $H_{c}$ to $H_{r}^{1}$, $H_{l}^{1}, H_{r}^{2}$, and $H_{l}^{2}$.

All treelike vascular structures can be processed using this set of rules. The recursive algorithm finishes with a hierarchy of enclosing vessel hulls where the root hull contains the entire vessel tree. A detailed description of each assembling case in abstract notation is given in Figure 7.

\section{Layout definition}

The decision of the vessel hull ordering in case 2 has a significant impact on the layout of the displayed vascular tree. Two different approaches have been investigated (see Figure 8). The adaptive layout is a left-to-right ordering based on the spatial relations of the vascular tree according to the currently used viewing direction. This results in less distortion at the cost of discontinuities during rotational interaction. In contrast to that a fixed layout is independent of the viewing direction. For clinical routine a standardized ordering of the blood vessels might be a reasonable solution. 


\section{Case 1:}

$H_{c} \quad \leftarrow P_{0}$

$\mathbf{v}_{r} \quad \leftarrow P_{j}-H_{c},\left\{P_{j} \mid P_{j}, \ell \in P \wedge \forall \ell\left(\ell\right.\right.$ left of $\left.\left.\overrightarrow{H_{c} P_{j}}\right)\right\}$

$\mathbf{v}_{l} \leftarrow P_{i}-H_{c},\left\{P_{i} \mid P_{i}, \ell \in P \wedge \forall \ell\left(\ell\right.\right.$ right of ${\overrightarrow{H_{c} P_{i}}}_{i})\}$

rad $\leftarrow\left|P_{n-1}-P_{0}\right|$

$H_{r} \quad \leftarrow H_{c}+\mathrm{rad} \cdot\left(\Re_{\varepsilon} \star \mathbf{v}_{r} /\left|\mathbf{v}_{r}\right|\right)$

$H_{l} \leftarrow H_{c}+\operatorname{rad} \cdot\left(\Re_{-\varepsilon} \star \mathbf{v}_{l} /\left|\mathbf{v}_{l}\right|\right)$

Case 2:

$H_{c} \leftarrow H_{c}^{1}$

$\left(V^{l}, V^{r}\right) \leftarrow$ if (changeOrder) then $\left(V^{2}, V^{1}\right)$ else $\left(V^{1}, V^{2}\right)$

$\Re_{H_{c}^{r}} \leftarrow \operatorname{RotationMatrix}\left(H_{c},-0.5 \max \left(\varangle\left(\mathbf{v}_{l}^{r}, \mathbf{v}_{r}^{l}\right), 0\right)\right)$

$\Re_{H_{c}^{l}}^{{ }^{l}} \leftarrow$ RotationMatrix $\left(H_{c},+0.5 \max \left(\varangle\left(\mathbf{v}_{l}^{r}, \mathbf{v}_{r}^{l}\right), 0\right)\right)$

$\mathbf{v}_{r} \leftarrow \mathbf{v}_{r}^{r}$

$\mathbf{v}_{l} \leftarrow \mathbf{v}_{l}^{l}$

$\mathrm{rad} \leftarrow \max \left(\mathrm{rad}^{l}, \mathrm{rad}^{r}\right)$

$H_{r} \quad \leftarrow H_{c}+\operatorname{rad} \cdot\left(\Re_{H_{r}} \star \mathbf{v}_{r} /\left|\mathbf{v}_{r}\right|\right)$

$H_{l} \quad \leftarrow H_{c}+\mathrm{rad} \cdot\left(\Re_{H_{\text {left }}} \star \mathbf{v}_{\text {left }} /\left|\mathbf{v}_{l}\right|\right)$

Case 3:

$H_{c} \leftarrow H_{c}^{1}$

$\mathbf{v}_{r} \leftarrow$ if $\left(H_{r}^{2}\right.$ left of $\left.\overrightarrow{H_{c} H_{r}^{1}}\right)$ then $H_{r}^{1}-H_{c}$ else $H_{r}^{2}-H_{c}$

$\mathbf{v}_{l} \leftarrow$ if $\left(H_{l}^{2}\right.$ right of $\left.\overrightarrow{H_{c} H_{l}^{1}}\right)$ then $H_{l}^{1}-H_{c}$ else $H_{l}^{2}-H_{c}$

$\mathrm{rad} \leftarrow \max \left(\operatorname{rad}^{1},\left|H_{c}-H_{r}^{2}\right|,\left|H_{c}-H_{l}^{2}\right|\right)$

$H_{r} \quad \leftarrow H_{c}+\mathrm{rad} \cdot \mathbf{v}_{r} /\left|\mathbf{v}_{r}\right|$

$H_{l} \quad \leftarrow H_{c}+\mathrm{rad} \cdot \mathbf{v}_{l} /\left|\mathbf{v}_{l}\right|$

Fig. 7. Assembling of vessel hulls.

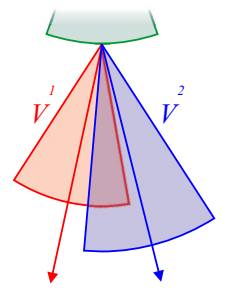

$0^{\circ}$ coronal

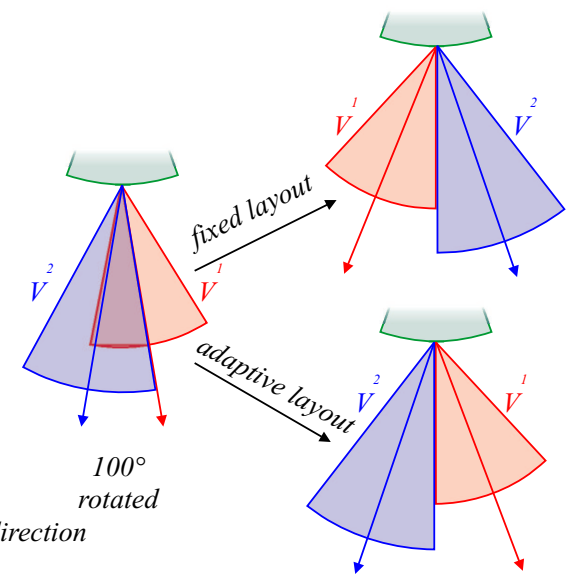

Fig. 8. Different layout definition 


\section{Image space partitioning}

Bevor rendering the final image the image space has to be partitioned into fragments for each vascular structure. A principle drawing direction is associated with each vessel segment. Each point of the projected vessel centerline maintains a scanline deduced from this principal drawing direction. These scanlines are transformed according to the rotational matrix $\mathfrak{R}_{H_{c}}$ (see Figure 9). The scanlines represent those parts of the image into which the corresponding re-sampled data from the dataset are rendered.

In order to avoid overlapping areas an appropriate start and endpoint for each scanline has to be determined. A directional distance map is defined by the projected centerlines of the vessel tree and its scanlines. In contrast to the traditional distance map the distance metric is not defined by an Euclidean distance but by a distance along scanlines. The result of this operation is a fragmented image space where each vessel segment is assigned a maximal image area.
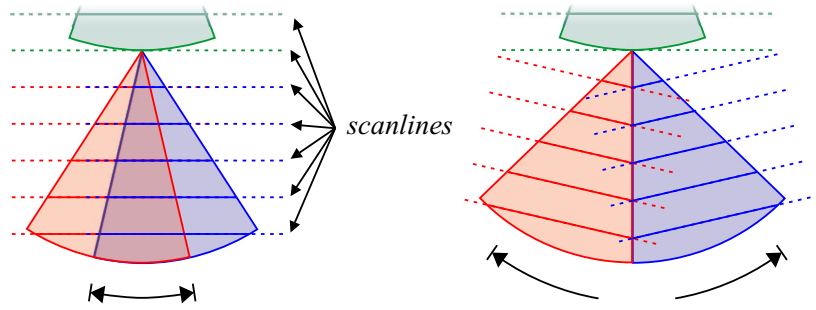

Fig. 9. Image space partitioning

\section{Results}

Table 1 summarizes the introduced methods: projected CPR (Proj.), stretched CPR (Stre.), straightened CPR (Stra.), and helical CPR (Helical.), multi-path projected CPR (M-Proj.), multi-path stretched CPR (M-Stre.), and untangled CPR (Untang.). The methods are grouped according to wether displaying a whole vessel tree is possible or not (Vessel tree). The criterion Spatial Perception indicates how radiologists judge the spatial expressiveness (i.e. the easiness to map positions within the CPR to locations in the volumetric dataset without computational aid). Wether the method preserves true distances in close vicinity of the computed centerline is depicted by the field Isometry. The possible occurrence of bones superimposing the tracked vessel is expressed by Occlusions (bone). The criterion Occlusions (artery) shows wether arteries may cross in image space and consequently overlap each other. Finally Rotation needed indicates if multiple viewing directions are needed in order to investigate the total vessel lumen. 


\begin{tabular}{l|c|c|c|c|c|c|c} 
& Proj. & Stre. & Stra. & Helical & M-Proj. & M-Stre. & Untang. \\
\hline Vessel tree & no & no & no & no & yes & yes & yes \\
\hline Spatial Perception & high & med & low & low & high & med & med \\
\hline Isometry & no & yes & yes & yes & no & yes & yes \\
\hline Occlusions of bones & poss. & no & no & no & poss. & no & no \\
\hline Occlusions of arteries & poss. & no & no & no & poss. & poss. & no \\
\hline Rotation needed & yes & yes & yes & no & yes & yes & yes \\
\hline
\end{tabular}

Table 1. Comparison of CPR methods.

\subsection{Single Vessel CPR Methods}

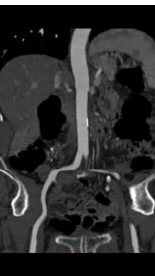

(a)

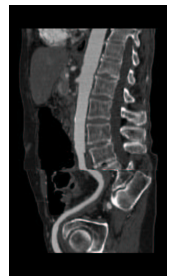

(b)

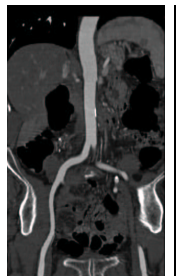

(c)

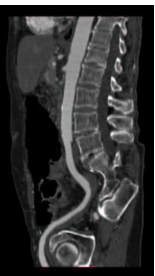

(d)

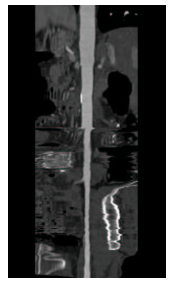

(e)

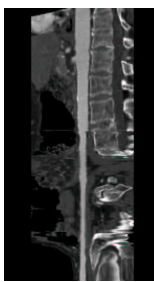

(f)

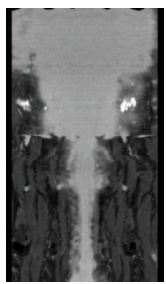

(g)

Fig. 10. Comparison of CPR methods: A coronal and sagittal display of a projected CPR $(a, b)$, stretched CPR (c,d), and straightened CPR (e,f). A helical CPR (g).
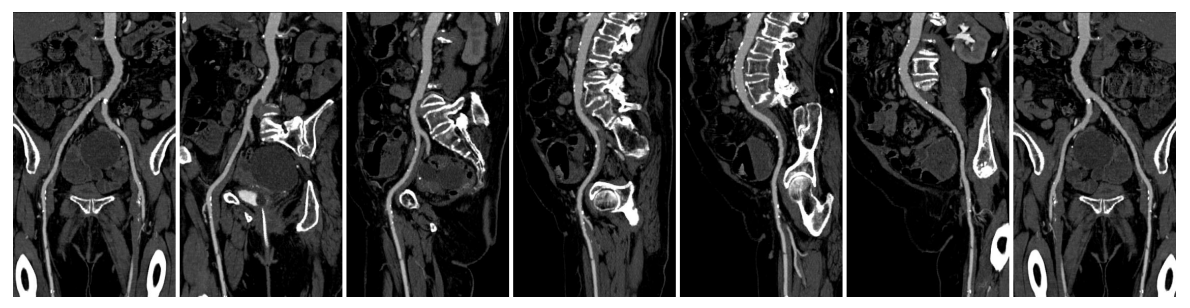

Fig. 11. Rotating stretched CPR: $0^{\circ}, 30^{\circ}, 60^{\circ}, 90^{\circ}, 120^{\circ}, 150^{\circ}, 180^{\circ}$.

A summary of the four different CPR methods displaying single vessel segments is presented in this Section. The comparison is performed on a dataset of the abdominal area (see Figure 10) as well as from peripheral arteries (see Figure 11 and Figure 12). 


\section{Projected CPR}

In Figure 10a,b a projected CPR from coronal and sagittal view is displayed. Projection artifacts arise whenever the vessel is in plane with the viewing direction.

\section{Stretched CPR}

Accordingly Figure 10c,d presents a stretched CPR. The vessel's isometry is preserved in the image.

\section{Straightened CPR}

In the case of a straightened CPR the vessel is straightened to a line centered in the image space (see Figure 10e,f).

\section{Helical CPR}

The application of a helical CPR technique is presented in Figure 10. A dataset with more anatomical features is presented in Figure 12. A constant angle and constant arc-length sampled helical CPR is compared to a straightened CPR image. The white arrows illustrate an example where the helical CPR outperforms a traditional CPR. The small flow-channel of the stenosis is not touched by the displayed longitudinal section of the straightened CPR and therefore not visible. However in both helical images this flow-channel is displayed. As eccentric lesions cause repetitive patterns in image space, the attention of the observer is immediately drawn to those areas even if a lesion is not visible in a standard CPR display.

\subsection{Vessel Tree CPR Methods}

This section presents the application of the introduced CPR methods. Vessel trees from a real world data set with a scanned resolution of 512x512x 988 are visualized.

\section{Projected Multi-Path CPR}

A projected multi-path CPR is presented in Figure 13. The projected multi-path CPR on the left provides an overview of the processed data set. A close-up of the upper image region is shown from different viewing directions.

\section{Stretched Multi-Path CPR}

If the vessel tree is mapped into the image space according to the stretched CPR method a combination of isometry preserving vessel segments is displayed. In Figure 14 the corresponding area was processed using the stretched multi-path CPR method. 


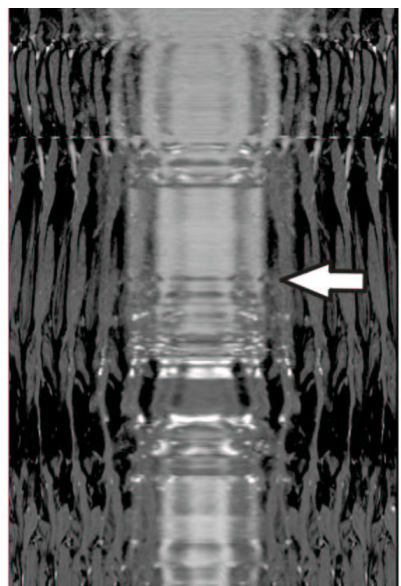

(a)

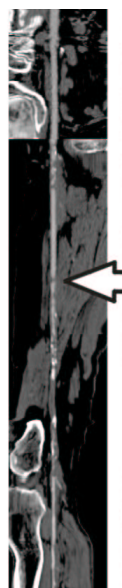

(b)

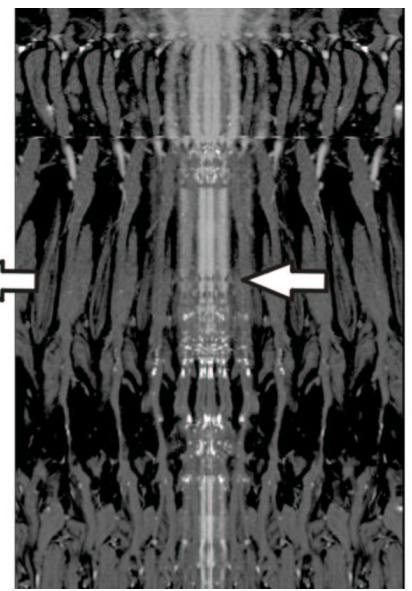

(c)

Fig. 12. A helical CPR with constant angle sampling (a), a straightened CPR (b), and constant arc-length sampled helical CPR (c).

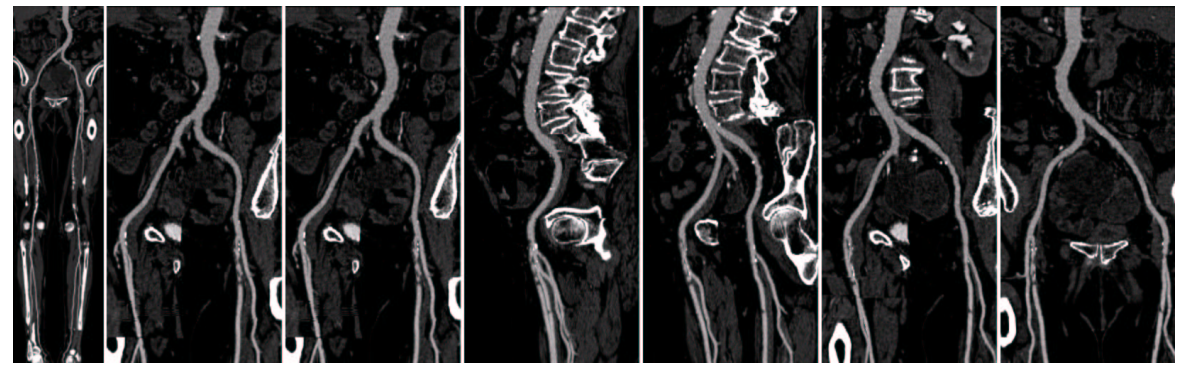

Fig. 13. Overview of a projected multi-path $\mathrm{CPR}$ at $0^{\circ}$. Enlargement of the projected multipath CPRs at $30^{\circ}, 60^{\circ}, 90^{\circ}, 120^{\circ}, 150^{\circ}, 180^{\circ}$.

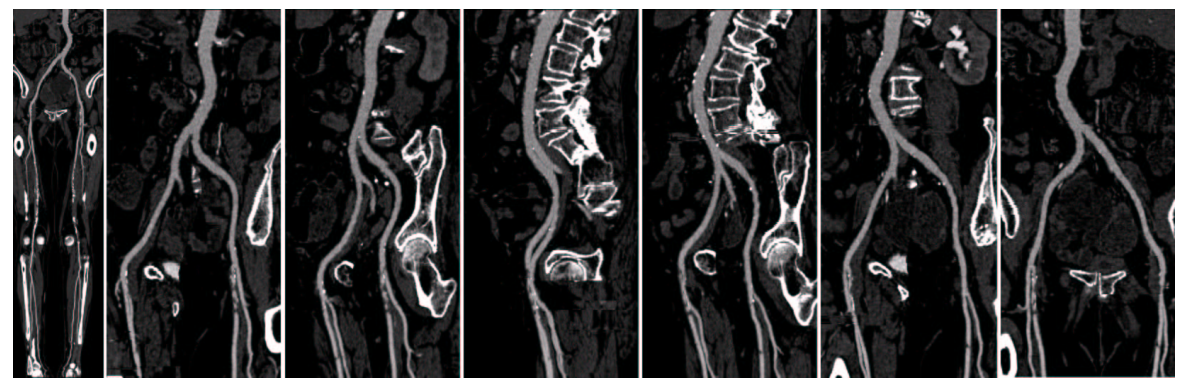

Fig. 14. Overview of a stretched multi-path $C P R$ at $0^{\circ}$. Enlargements of the stretched multipath CPRs at $30^{\circ}, 60^{\circ}, 90^{\circ}, 120^{\circ}, 150^{\circ}, 180^{\circ}$. 


\section{Untangled CPR}

A comparison of a stretched multi-path CPR and an untangled CPR is presented in Figure 15. In the case of a lateral view the multi-path CPR display provides hardly any diagnostically relevant information. Many superimposed arteries obscure each other. In comparison to that the untangled CPR still provides an unobstructed view of the entire vascular tree. Each vessel segment is displayed in diagnostic quality.

The examination is intended to be done on just a small set of pre-computed images, the performance of the algorithm is acceptable for applications in the clinical workflow. The displayed untangled CPR image in Figure 15 was calculated with an original image size of $1164 \times 1097$ pixels. The average rendering time per image of the current Java based implementation on a PC workstation with an Intel PIII $1 \mathrm{GHz}$ main processor takes 2.3 seconds.

Figure 16 presents a sequence of untangled CPR images from an abdominal CTangiographic dataset. A fixed layout was used. A 1D transfer function was applied to the re-sampled data approximating the tissue color of the anatomical structures.

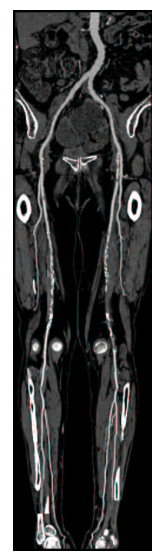

(a)

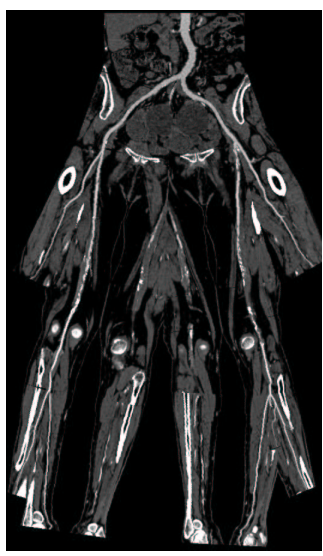

(b)

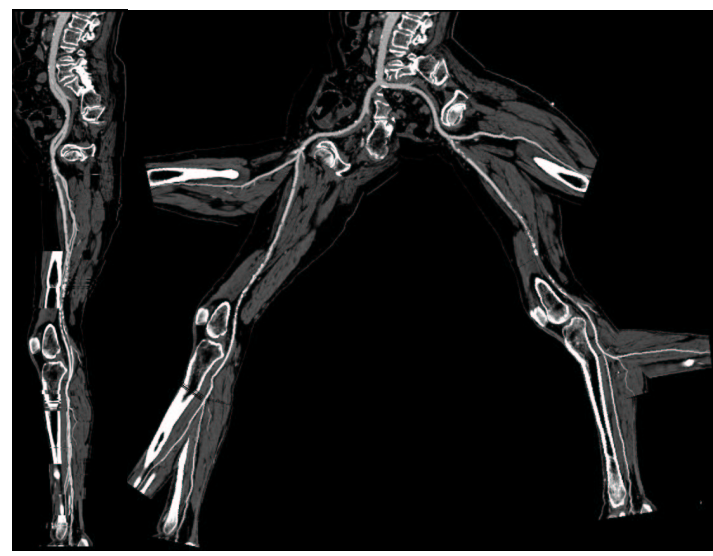

(c) (d)

Fig. 15. A peripheral CTA dataset rendered from coronal and sagittal view using stretched multi-path CPR (a, c) and untangled CPR (b, d) respectively (fixed layout)

\section{Conclusions}

In this paper methods for the generation of Curved Planar Reformation (CPR) images have been presented. This method allows the visualization of entire tubular structures with minimal modification of the original data. The main application of this visualization method is Computed Tomography Angiography (CTA). With volume rendering even mild vessel wall calcifications may obscure the true vessel lumen 


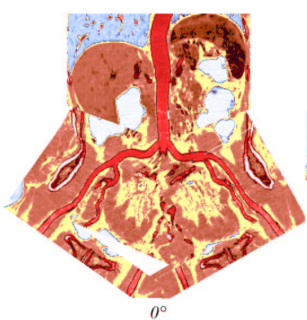

$0^{\circ}$

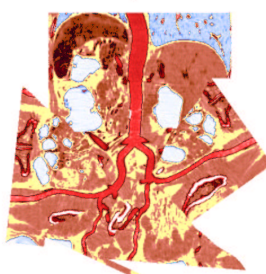

$180^{\circ}$

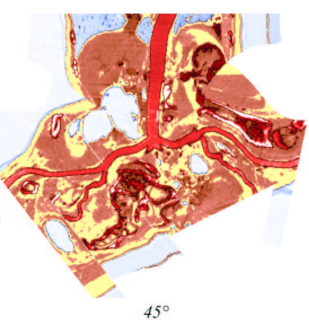

$45^{\circ}$

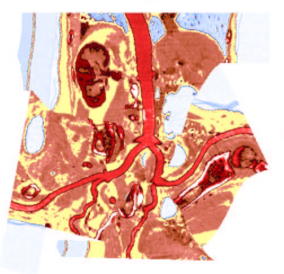

$225^{\circ}$

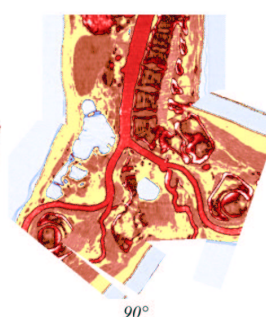

$90^{\circ}$

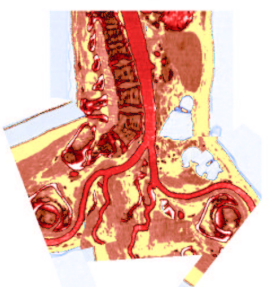

$270^{\circ}$

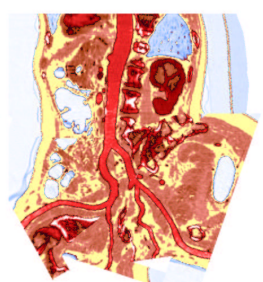

$135^{\circ}$

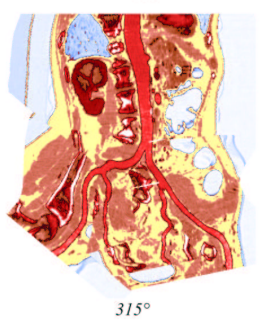

Fig. 16. A colored sequence of untangled CPR images from different viewing directions.

(flow channel), which is the clinically relevant information. CPR displays the vessel lumen also in the presence of vessel wall calcifications.

Four different methods (i.e., projected, stretched, straightened, and helical CPR) have been demonstrated. A comparison of the three methods with respect to spatial perception, isometry, and possible occlusions has shown that the stretched CPR is the preferred method for many applications. With the helical CPR the dataset is re-sampled in a spiral manner making the entire vessel visible. The motivation for this visualization technique was not an imitation of the natural appearance of the object but the revelation of diseased vessel segments. An explorative study has shown that the detection of vessel abnormalities is possible from such visualizations which literally peel-off the vessel volume.

As fewer images have to be generated and as the spatial relationships of the vascular tree are well preserved, we believe that multi-path CPRs are not only very well suited for diagnostic purposes, but also for the documentation and for communicating the extent of diseases to the treating physician. However, this method suffers from arteries possibly crossing each other in image space and thus reduce the diagnostic value of the image.

Small distortions of the vascular tree prevent self-intersections in the case of the untangled CPR. The untangled CPR has significant advantages over existing multipath CPR techniques. This new technique produces an unobscured display of a vascular tree, independent of the viewing direction. Small rotations around the branching points of a vessel tree eliminate occlusions. Therefore the size of the introduced distortion is kept small.

Even though the use of image space is not optimal, the main requirement of unobscured display of vessels from any viewing direction is fulfilled. In addition, 
untangled CPR preserves isometry which is an important requirement for vascular lesion assessment. The potential for clinical application of this technique is obvious. The untangled CPR provides a more efficient way to assess any complex arterial trees for the presence and extent of vascular disease. Clinical validity and applicability to other vascular territories are currently investigated.

\section{Acknowledgements}

The work presented in this publication has been funded by the ADAPT project (FFF804544). ADAPT is supported by Tiani Medgraph, Vienna (http://www.tiani.com), and the Forschungsförderungsfonds für die gewerbliche Wirtschaft, Austria.

See http://www.cg.tuwien.ac.at/research/vis/adapt for further information on this project.

\section{References}

1. Kanitsar, A., Wegenkittl, R., Felkel, P., Fleischmann, D., Sandner, D., Gröller, E.: Computed Tomography Angiography: A Case Study of Peripheral Vessel Investigation. In: IEEE Visualization 2001. (2001) 477-480

2. Wink, O., Niessen, W., Viergever, M.: Fast Delineation and Visualization of Vessels in 3-d Angiographic Images. IEEE Transactions on Medical Imaging 19 (2000) 337-346

3. Zahlten, C., Juergens, H., Peitgen, H.O.: Reconstruction of Branching Blood Vessels from CT-Data. In: Eurographics Workshop on Visualization in Scientific Computing. (1994) 161-168

4. Avants, B., Williams, J.: An Adaptive Minimal Path Generation Technique for Vessel Tracking in CTA/CE-MRA Volume Images. In: MICCAI 2001. (2000) 707-716

5. He, S., Dai, R., Lu, B., Cao, C., Bai, H., Jing, B.: Medial Axis Reformation: A New Visualization Method for CT Angiography. Academic Radiology 8 (2001) 726-733

6. Felkel, P., Fuhrmann, A., Kanitsar, A., Wegenkittl, R.: Surface Reconstruction Of The Branching Vessels For Augmented Reality Aided Surgery. In: BIOSIGNAL 2002. (2002) 252-254

7. Hahn, H., Preim, B., Selle, D., Peitgen, H.O.: Visualization and Interaction Techniques for the Exploration of Vascular Structures. In: IEEE Visualization 2001, ACM (2001) 395-402

8. Kanitsar, A., Fleischmann, D., Wegenkittl, R., Felkel, P., Gröller, M.E.: CPR - Curved Planar Reformation. In: IEEE Visualization 2002. (2002) 37-44

9. Addis, K., Hopper, K., Iyriboz, T., Liu, Y., Wise, S., Kasales, C., Blebea, J., Mauger, D.: CT Angiography: In Vitro Comparison of Five Reconstruction Methods. In: Am. J. Roentgenol. (2001) 177:1171-1176

10. Achenbach, S., Moshage, W., Ropers, D., Bachmann, K.: Curved Multiplanar Reconstructions for the Evaluation of Contrast-Enhanced Electron-Beam CT of the Coronary Arteries. In: Am. J. Roentgenol. (1998) 895-899

11. Koechl, A., Kanitsar, A., Lomoschitz, F., Groeller, E., Fleischmann, D.: Comprehensive Assessment of Peripheral Arteries using Multi-path Curved Planar Reformation of CTA Datasets. In: Europ. Rad. Volume 13. (2003) 268-269 
12. Rubin, G.D., Schmidt, A., Logan, L., Sofilos, M.: Multi-Detector Row CT Angiography of Lower Extremity Arterial Inflow and Runoff: Initial Experience. In: Radiology 2001. (2001) 146-158

13. Klok, F.: Two Moving Coordinate Frames for Sweeping along a 3D Trajectory. In: Computer Aided Geometry Design. (1986) 3:217-229

14. Kanitsar, A., Wegenkittl, R., Fleischmann, D., Gröller, M.E.: Advanced Curved Planar Reformation: Flattening of Vascular Structures. In: IEEE Visualization 2003. (2003) 4350 